\title{
Extension of Fock Theory for Currents in the Penumbra Region ${ }^{1}$
}

\author{
V. H. Weston ${ }^{2}$ \\ Conductron Corporation, Ann Arbor, Mich.
}

(Received Oct. 26, 1964; revised Feb. 1, 1965)

\begin{abstract}
For high-frequency scattering by a plane electromagnetic wave incident upon a convex surface, the current distribution in the transition region between the illuminated and shadow region, and beyond, was considered by V. A. Fock. The effect of the surface being flat and no longer parabolic in the penumbra region is considered here. There are two cases considered, one for which a plane wave is incident along a plane perfectly conducting surface towards a drop in this surface, this drop represented by a locally convex parabolic cylinder smoothly joined to the plane section. An expression is derived for the fields on the convex surface in the penumbra region, and deep in the shadow region the appropriate creeping wave expression is obtained. For the other case, a plane wave is incident towards a convex parabolic section smoothly joined to a planar section with the parabolic section lying in the illuminated region, and the direction of the incident wave being along the planar section. It is shown that far along this flat section, the total field on the surface is comprised of the incident field plus a traveling wave field which decreases as the square root of the distance from the illuminated side.
\end{abstract}

\section{Introduction}

For high-frequency scattering by a plane electromagnetic wave incident upon a smooth convex (locally parabolic) surface, the current distribution in the transition region between the illuminated and shadow regions was obtained by V. A. Fock [1946]. Fock gave, as an estimate of the width of the penumbra region, the following relation

$$
d \sim\left(\lambda R^{2} / \pi\right)^{1 / 3}
$$

where $R$ is the radius of curvature at the geometric optics shadow boundary. The current distribution was expressed in terms of a contour integral involving Airy integrals, and which, asymptotically, gave the physical optics current distribution in the illuminated region and a creeping wave type of current distribution in the shadow region.

The purpose of this paper is to extend the results to the case where the geometry of the surface is cylindrical and is such that over a portion large compared to wavelength, the surface is approximated by a flat section smoothly joined to a parabolic section. Hence the surface, besides possessing a region of infinite radius of curvature, also possesses a discontinuity in curvature, i.e., a discontinuity in the second and all higher derivatives of the surface profile. The incident field is assumed to be a plane wave traveling along in a direction parallel to the plane of the flat portion, and thus the shadow boundary and join coincide.

There are two cases treated. First considered is the case where the plane wave is propagating along the flat portion towards and normal to the junction of the flat section with the parabolic section. The convex parabolic section is thus in the geometric optics shadow zone. Next considered is the case where the incident wave is traveling in the opposite direction such that the parabolic section is in the geometric optics illuminated region.

The analysis is restricted to the important case where the polarization of the electromagnetic field is perpendicular to the surface. Hence since, in addition, the geometry is cylindrical, the

${ }^{1}$ The research reported in this paper was sponsored in part by Air Force Ballistic Systems Division under Contract No. AF 04(694)-597 and by Advanced Research Projects Agency under ARPA Order No. 558

${ }^{2}$ Present address, University of Michigan, Ann Arbor, Mich. 
problem reduces to a scalar problem. A briet discussion taking into account the other polarization and a more general noncylindrical surface is given at the end. Briefly, the analysis will proceed as follows: The unknown field distribution on the surface will be expressed in terms of an integral equation which is then simplified by the following process. Physical postulates are placed upon the behavior of the unknown field distribution in the limit of small wavelengths. A highfrequency asymptotic analysis then reduces the integral equation to a simplified form which is amenable to solution with employment of the Laplace transform. The field distribution is easily obtained and in the first case considered, is expressed in the form of a contour integral involving Airy integrals. Deep in the shadow region this integral formulation is then expressible in terms of a sum of residues, physically representing the phenomenon of creeping waves. In the second case considered, it is shown that on the flat section, a long distance from the junction, the field is comprised of the incident wave plus a traveling wave whose magnitude decreases as the square root of the distance of the field point from the join.

\section{Convex Section in the Shadow Region}

A cartesian coordinate system will be used and oriented such that the $z$-axis lies along the junction of the plan and parabolic sections, and the plane $x=0, y<0$ describes the flat portion of the surface. The convex section will be in the half-space $y>0$, and will be represented locally in the neighborhood of the junction by the parabolic cylinder

$$
x=-\frac{1}{2 R} y^{2}
$$

where $R$ is the radius of curvature of the parabolic section at the junction. Since the analysis will be concerned with the neighborhood of the junction, the curvature of the convex surface will not be prescribed for large $y$.

A plane wave will be considered incident along the plane section towards, and normal to the junction $(x=0, y=0)$. The wave will be polarized perpendicular to the conducting surface, with the magnetic intensity in the $z$ direction. The incident field has the prescribed form

$$
\begin{aligned}
& \mathbf{H}=\mathbf{i}_{z} e^{i k y} \\
& \mathbf{E}=-\mathbf{i}_{x} e^{i k y} \sqrt{\mu_{0} / \epsilon_{0}}
\end{aligned}
$$

where harmonic time dependence $e^{-i \omega t}$ is assumed. The problem reduces to that of a scalar problem involving the magnetic field component, which must satisfy the following integral equation on the surface [Maue, 1949]:

$$
\frac{\partial u}{\partial n}=0, u(P)=2 u_{0}(P)+\frac{1}{2 \pi} \int \frac{\partial G}{\partial n_{Q}} U(Q) d s_{Q}
$$

with

$$
G=\frac{e^{i k \rho}}{\rho} \text { and } \rho=\left|\mathbf{x}_{P}-\mathbf{x}_{Q}\right|
$$

Since the source of illumination is on the surface, Maue's equation implies that

$$
2 u_{0}=e^{i k y} .
$$

Using the results of (9), (10), and (11) of [Weston, 1962], and therein setting $a_{1}=0$, and $a_{2}=1 / R$, (2) reduces to

$$
u(y)=e^{i k y}-\frac{i k}{2} \int_{-\infty}^{\infty} d t \frac{u(t)}{r} H_{1}^{(1)}(k r) w(t, y)
$$


where

$$
\begin{aligned}
& r=\left[(y-t)^{2}+\frac{1}{4}\left(\tilde{a} t^{2}-a y^{2}\right)^{2}\right]^{1 / 2} \\
& w(t, y)=\tilde{a} t(t-y)-\frac{1}{2}\left(\tilde{a} t^{2}-a y^{2}\right)
\end{aligned}
$$

with the relationships

$$
\begin{array}{llll}
t<0, & \tilde{a}=0, & t>0, & \tilde{a}=R^{-1} ; \\
y<0, & a=0, & y>0, & a=R^{-1} .
\end{array}
$$

To simplify the analysis and to reduce the integral equation to a form amenable for solution the following physical postulates will be placed upon the behavior of the unknown surface field $u(y)$. On the plane section the total field will be comprised of the incident wave plus a wave reflected by the junction, as indicated by the following relationship:

$$
y<0, u(y)=e^{i k y}+e^{-i k y} I_{-}(y) .
$$

In addition in the high-frequency limit, it will be assumed that the factor $I_{-}(y)$ is a slowly varying function compared to the oscillatory factor $\exp \{-i k y\}$. On the parabolic section the field is a wave traveling away from the junction, having the form

$$
y>0, u(y)=e^{i k s} I_{+}(y),
$$

with

$$
s=\int_{0}^{y}\left[1+y^{2} / R^{2}\right]^{1 / 2} d y
$$

being the arc-length along the surface, measured from the junction. Again it will be assumed that the factor $I_{+}(y)$ is a slowly varying function in comparison with the oscillatory factor. In the limit where $k R \rightarrow \infty$, the following must hold: $I_{-}(y) \rightarrow 0$ and $I_{+}(y) \rightarrow 1$.

From (3), (4), and (5) the following integral equation holding for $y<0$ is obtained

$$
e^{i k y} I_{-}(y)=-\frac{i k}{4 R} \int_{0}^{\infty} d t I_{+}(t) e^{i k s(t)} H_{1}^{(1)}(k r) \frac{\left(t^{2}-2 t y\right)}{r}
$$

with

$$
r^{2}=(y-t)^{2}+\frac{t^{4}}{4 R^{2}}
$$

for $y>0$

$$
\begin{aligned}
e^{i k s(y)} I_{+}(y)=e^{i k y}-\frac{i k y^{2}}{4 R} \int_{-\infty}^{0} \frac{d t}{r}\left[e^{i k t}+e^{-i k t} I_{-}(t)\right] H_{1}^{(1)}(k r) & \\
& -\frac{i k}{4 R} \int_{0}^{\infty} \frac{d t}{r} e^{i k s(t)} I_{+}(t) H_{1}^{(1)}(k r)(t-y)^{2}
\end{aligned}
$$


where

$$
r^{2}=(y-t)^{2}+\frac{y^{4}}{4 R^{2}}
$$

and

$$
r^{2}=(y-t)^{2}+\frac{\left(y^{2}-t^{2}\right)^{2}}{4 R^{2}}
$$

in the first and second integrals respectively.

Since $I_{+}(y)$ is a relatively slowly varying function, (9) can be written in the form

$$
I_{-}(y)=\frac{-i}{2 k R} \int_{0}^{\infty} \exp [i k f] v(t, y) d k t
$$

where $v(t, y)$ is a slowly varying function and

$$
f(t, y)=y+s(t)+r(y, t) .
$$

Since $k f(t, y)$ has no stationary point in the region of integration, the dominant part of the integral arises from the lower limit. Thus on integrating by parts one obtains

$$
I_{-}(y) \sim 0\left(\frac{1}{k R}\right) I_{+}(0) .
$$

But on taking the limit as $y \rightarrow 0+$ in (10) one obtains

$$
I_{+}(0) \sim 1
$$

thus $I_{-}(y)$ can be neglected, which is to be expected since the surfaces are smoothly joined.

For the region $y>0$, that portion of the integral in (10) that contains the reflected wave has no stationary point; hence its dominant part arises from the upper limit of integration. Since in addition the reflected wave is the order of $(1 / k R)$ of the incident wave, this integral is small compared to that portion of the integral in (10) containing the incident field. The dominant portion of the last integral in (10) arises from the region $0 \leqslant t \leqslant y$. The reason is that there exists a stationary point in the region $0 \leqslant t \leqslant y$, and no stationary point in the remaining interval $y<t<\infty$. Hence in the high-frequency limit, the integral over the range $y<t<\infty$ may be evaluated by integration by parts, the dominant portion arising from the lower limit of integration. Thus this integral is the order of $(k R)^{-1} I_{+}(y)$ and can be neglected compared to the left-hand side of (10).

With these considerations (10) can be approximated by

$$
e^{i k s} I_{+}(y)=e^{i k y}-\frac{i k y^{2}}{4 R} \int_{-\infty}^{0} d t \frac{e^{i k t}}{r} H_{1}^{(1)}(k r)-\frac{i k}{4 R} \int_{0}^{y} \frac{d t}{r} e^{i k s(t)} I_{+}(t) H_{1}^{(1)}(k r)(t-y)^{2}
$$

where the $r$ 's are specified accordingly by (11) and (12).

To obtain the appropriate asymptotic solution to (13) for large $k R$ we shall set

$$
\begin{gathered}
m=(k R)^{1 / 3} \\
k y=m^{2} \xi \\
k t=m^{2} \tau \\
I_{+}(y)=J(\xi) .
\end{gathered}
$$


Multiply both sides of (13) by the factor $\exp \{-i k s(y)\}$. In obtaining the appropriate asymptotic value of the resulting integral corresponding to the first integral on the right-hand side of (13) the following asymptotic expressions are used:

$$
\begin{aligned}
& k[y-s(y)] \sim-\frac{1}{6} \xi^{3}+0\left(\frac{1}{m^{2}}\right) \\
& k r \sim(\xi-\tau) m^{2}+\xi^{4} / 8(\xi-\tau)
\end{aligned}
$$

where $r$ is specified by (11). Hence in the limit $m \rightarrow \infty$, the following integral

$$
\frac{i k^{2} y}{4 R} \int_{-\infty}^{0} \frac{d t}{r} H_{1}^{(1)}(k r) e^{i k(t-y)}
$$

reduces to

$$
\sim \sqrt{\frac{2}{\pi}} \frac{\xi^{2}}{4} e^{-i \pi / 4} \int_{-\infty}^{0} \frac{\exp i\left\{\xi^{4} /[8(\xi-\tau)]\right\}}{(\xi-t)^{3 / 2}} d \tau \sim \frac{2}{\sqrt{\pi}} e^{-i \pi / 4} \int_{0}^{x} e^{i z^{2}} d z
$$

with

$$
X=\left(\frac{\xi}{2}\right)^{3 / 2}
$$

In evaluating the asymptotic value of the remaining integral, the following relations are used:

$$
\begin{gathered}
k r \sim(\xi-\tau) m^{2}+\frac{(\xi-\tau)(\xi+\tau)^{2}}{8} \\
k[s(t)-s(y)+r] \sim-\frac{(\xi-\tau)^{3}}{24}+0\left(\frac{1}{m^{2}}\right)
\end{gathered}
$$

where $r$ is specified by (12). Thus in the limit $m \rightarrow \infty$, the second integral given by

$$
\frac{-i k}{4 R} \int_{0}^{y} \frac{d t}{r} I_{+}(t) H_{1}^{(1)}(k r)(t-y)^{2} \exp \{i k[s(t)-s(y)]\}
$$

reduces to

$$
-\frac{e}{4}^{-i \pi / 4} \sqrt{\frac{2}{\pi}} \int_{0}^{\xi} d \tau(\xi-\tau)^{1 / 2} J(\tau) \exp \left[-i(\xi-\tau)^{3} / 24\right]+0\left(\frac{1}{m^{2}}\right)
$$

Using these results (13) reduces to the form

$J(\xi) \sim \exp \left(-i \xi^{3} / 6\right)\left[1-\frac{2}{\sqrt{\pi}} e^{-i \pi / 4} \int_{0}^{x} e^{i z^{2}} d z\right]-\frac{e^{-i \pi / 4}}{4} \sqrt{\frac{2}{\pi}}$

$$
\int_{0}^{\xi} d \tau(\xi-\tau)^{1 / 2} J(\tau) \exp \left[-i(\xi-\tau)^{3} / 24\right]
$$

This resulting equation is of a form which is amenable to solution by well known methods. As a preliminary the following Laplace transforms will be defined,

$$
\bar{J}(\mathrm{p})=\int_{0}^{\infty} e^{-p \xi} J(\xi) d \xi
$$




$$
\begin{aligned}
& \mathrm{M}(p)=\int_{0}^{\infty} e^{-p \xi} \xi^{1 / 2} \exp \left(-i \xi^{3} / 24\right) d \xi \\
& N(p)=\int_{0}^{\infty} \exp \left(-p \xi-i \xi^{3} / 6\right)\left[1-\frac{2}{\sqrt{\pi}} e^{-i \pi / 4} \int_{0}^{x} e^{i z^{2}} d z\right] d \xi .
\end{aligned}
$$

With these definitions, the solution to (22) is obtained by taking the Laplace transforms of both sides, yielding

$$
\bar{J}(p)=N(p)-\frac{e}{4}^{-i \pi / 4} \sqrt{\frac{2}{\pi}} M(p) \bar{J}(p) .
$$

Rearranging the terms gives the resulting expression for $\bar{J}(p)$,

$$
\bar{J}(p)=N(p) /\left\{1+\frac{e}{4}^{-i \pi / 4} \sqrt{\frac{2}{\pi}} M(p)\right\}^{-1} .
$$

Fortunately $M(p)$ has already been evaluated [Weston, 1962] and has the form

$$
M(p)=2 \pi \sqrt{\pi 8}\left[A_{i}\left(A_{i}^{\prime}-i B_{i}^{\prime}\right)+\frac{i}{2 \pi}\right] e^{i 3 \pi / 4}
$$

where $A_{i}$ and $B_{i}$ are Airy integrals [Miller, 1946], with argument-ip2 $2^{1 / 3}$. The denominator of (27) may be simplified by using the Wronskian relationship of the Airy integrals, becoming

$$
2 \pi i A_{i}\left(A_{i}^{\prime}-i B_{i}^{\prime}\right)
$$

Unfortunately $N(p)$ has to be computed; for purposes of evaluation, the limits of integration over the $z$ variable in (25) will be changed from 0 and $x$ to $x$ and $\infty$, where in doing so use is made of the relationship

$$
\int_{0}^{\infty} e^{i z^{2}} d z=\sqrt{\frac{\pi}{2}} e^{i \pi / 4}
$$

$N(p)$ reduces to the form

$$
N(p)=\frac{2}{\sqrt{\pi}} e^{-i \pi / 4} F(p)
$$

with

$$
F(p)=\int_{0}^{\infty} \exp \left(-p \xi-i \xi^{3} / 6\right) \int_{x}^{\infty} \exp \left(i z^{2}\right) d z d \xi
$$

Expression $F(p)$ is evaluated explicitly in the appendix.

An explicit expression can now be obtained for $\bar{J}(p)$. From (27), (29), (30), and (A.21) (in the appendix) the following expression is obtained:

$$
\left.\bar{J}(p)=-i 2^{4 / 3} \frac{\left[1 / 3+1 / 2 \int_{0}^{q}\left(A_{i}(x)-i B_{i}(x)\right]\right.}{A_{i}^{\prime}(q)-i B_{i}^{\prime}(q)} d x\right]
$$


where

$$
q=-i p 2^{1 / 3}
$$

Now $J(p)$ can be obtained by taking the inverse transform yielding

$$
J(\xi)=\frac{1}{2 \pi i} \int_{\mathrm{c}-i \infty}^{\mathrm{c}+i_{\infty}} e_{\stackrel{\xi}{\xi} \bar{J}}(p) d p .
$$

Changing the variable of integration by setting $p=i t 2^{-1 / 3}$ and using the Airy integral notation used by Fock [1946] given as follows,

$$
w_{1}(t)=i \sqrt{\pi}\left[A_{i}(t)-i B_{i}(t)\right]
$$

one obtains the following expression for the field on the surface

$$
u(y)=\frac{1}{2 \pi i} \int_{-\infty-i c}^{\infty-i c} \frac{h(t)}{w_{1}^{\prime}(t)} \exp \{i k s+i y t / d\} d t,
$$

where

and

$$
h(t)=2 i \sqrt{\pi / 3}+\int_{0}^{t} w_{1}(x) d x
$$

$$
d=\frac{m^{2} 2^{1 / 3}}{k}=\left(\frac{\lambda R^{2}}{\pi}\right)^{1 / 3}
$$

The parameter $d$ is the distance that Fock [1946] gives for an estimate of the width of the penumbra region produced by a plane wave incident upon a parabolic surface. For the case at hand the parameter $d$ may be thought of as being an estimate of the width of the transition region. For $y$ sufficiently large ( $y$ the order of $d$ ), the integral representation may be expressed in terms of the residues at the zeros of $w_{1}^{\prime}(t)$, giving the result expressing the field in terms of the creeping waves

$$
u(y)=e^{i k s} \sum_{l=1}^{\infty} C_{l} \exp \left[i y t_{l} / d\right]
$$

where

$$
C_{l}=\left[2 i \sqrt{\pi / 3}+\int_{0}^{t_{l}} w_{1}(x) d x\right] /\left[t_{l} w_{1}\left(t_{l}\right)\right]
$$

with $t_{l}$ being the roots of $w_{1}^{\prime}(t)=0$.

For computational purposes, it is best to use the following relationship involving the Airy integrals:

$$
w_{1}(t)=e^{\pi i / 6} 2 \sqrt{\pi} A_{i}\left(t e^{+2 \pi i / 3}\right) .
$$

Setting $t_{l}=e^{i \pi / 3} \beta_{l}, \quad$ (38) and (39) become

$$
u(y)=\sum_{l=1}^{\infty} C_{l} \exp \left\{i k s+i y \beta_{l} e^{i \pi / 3} / d\right\}
$$

with

$$
C_{l}=\left[1 / 3+\int_{0}^{\beta_{l}} A_{i}(-x) d x\right] /\left[\beta_{l} A_{i}\left(-\beta_{l}\right)\right] .
$$


The values of $\beta_{l}$ (the roots of $\left.A_{i}^{\prime}(-\beta)=0\right)$ and $A_{i}\left(-\beta_{l}\right)$ can be obtained from tables given by Logan [1959]. The integrals $\int_{0}^{\beta} A_{i}(-x) d x$ can then be interpolated from the National Bureau of Standards Tables [1958]. Proceeding in this manner one can easily compute the coefficients $C_{l}$. Particular values of the appropriate coefficient involved are given in table 1 for the first three roots $(l=1,2$, and 3$)$.

TABLE 1. Roots and residue coefficients

\begin{tabular}{r|r|r|r|r}
\hline \hline$l$ & $\beta_{l}$ & $A_{i}\left(-\beta_{l}\right)$ & $\int_{0}^{\beta_{l}} A_{i}(-x) d x$ & \multicolumn{1}{c}{$C_{l}$} \\
\hline & & & & \multicolumn{1}{|c}{} \\
\hline & 1.0188 & 0.5357 & 0.4757 & 1.4823 \\
3 & 3.2482 & -.4190 & .7008 & -0.7598 \\
\hline & 4.8201 & .3804 & .6511 & .5369 \\
\hline
\end{tabular}

Away from the locally parabolic section the field on the surface given by (41) may be generalized to include a general convex surface of varying curvature by using the fact that $C_{l}$ are the launching factors of the creeping waves. Thus using the general expression for the creeping waves [Keller and Levy, 1959] one has

$$
u(s)=\left[\frac{\kappa(s)^{1 / 6}}{\kappa(0)}\right] \sum_{l=1} C_{l} \exp i\left\{k s+e^{i \pi / 3} \beta_{l}\left(\frac{k}{2}\right)^{1 / 3} \int_{0}^{s} \kappa^{2 / 3} d s\right\}
$$

where $\kappa(s)$ is the curvature at a distance $s$ along the surface measured from the $z$-axis.

\section{Convex Section in the Illuminated Region}

Having considered the case where the incident wave is propagating along the planar section with the parabolic section in the shadow region, the case where the plane wave is incident to the planar section with the parabolic section in the illuminated region, will be briefly considered. The same coordinate system and surface will be used as in the part 2 , but the incident plane wave will have the form

$$
\begin{aligned}
& \mathbf{H}=\hat{\mathbf{i}}_{z} e^{-i k y} \\
& \mathbf{E}=\hat{\mathbf{i}}_{x} e^{-i k y} \sqrt{\mu_{0} / \epsilon_{0}} .
\end{aligned}
$$

Again (2) will be used to describe the total magnetic field on the surface, but with

$$
u_{0}(p)=e^{-i k y} .
$$

The fields on the surface will be assumed to have the general form

$$
\begin{gathered}
y>0 \quad u(y)=e^{-i k y} I_{+}(y)+e^{i k s} K_{+}(y) \\
y<0 \quad u(y)=e^{-i k y} I_{-}(y)
\end{gathered}
$$

where the second term in (44) is the wave reflected at the junction. Since the surface is smoothly joined, this reflected wave is of lower order magnitude and to the first approximation can be neglected.

Using (3), but with the nonhomogeneous term $\exp (i k y)$ replaced by $2 \exp (-i k y)$, together with (4) and (5) and relations (44) and (45), one obtains the following, holding for $y<0$,

$$
I_{-}(y)=2-\frac{i k}{4 R} \int_{0}^{\infty} d t H_{1}^{(1)}(k r) \frac{\left(t^{2}-2 t y\right)}{r}\left\{I_{+}(t) e^{i k(y-t)}+K_{+}(t) e^{i k(y+s)}\right\}
$$


where $r$ is prescribed by (9a). Using the relationships given by (15) and (16), together with

$$
\begin{aligned}
& I_{-}(y)=J_{-}(\xi) \\
& I_{+}(y)=J_{+}(\xi),
\end{aligned}
$$

and taking the asymptotic limit as $m \rightarrow \infty$, (46) reduces to

$$
J_{-}(\xi)=2-\frac{e^{-i \pi / 4}}{4} \sqrt{\frac{2}{\pi}} \int_{0}^{\infty} d \tau \frac{\left(\tau^{2}-2 \tau \xi\right)}{(\tau-\xi)^{3 / 2}} \exp \left\{\frac{i \tau^{4}}{8(\tau-\xi)}\right\} J_{+}(\tau)\left[1+0\left(\frac{1}{m^{2}}\right)\right] .
$$

In a similar manner one can proceed and obtain the following integral equation holding for $y>0$,

$$
J_{+}(\xi)=2-\frac{e^{-i \pi / 4}}{4} \sqrt{\frac{2}{\pi}} \int_{\xi}^{\infty} d \tau J_{+}(\tau)(\tau-\xi)^{1 / 2} \exp \left\{\frac{i}{8}(\tau+\xi)^{2}(\tau-\xi)\right\}
$$

The solution to this equation at a point $\xi$ depends only on the values of the field at points in the illuminated region such that $\tau \geqslant \xi$, hence is independent of the planar section. Thus, as is expected, $J_{+}(\xi)$ is the solution that would be obtained if the complete surface were parabolic. Thus to a first approximation in $k R$, we have for $y>0$ from [Fock, 1946]

$$
J_{+}(\xi)=\frac{1}{\sqrt{\pi}} \int_{\Gamma_{1}} d t \frac{\exp \left[-i \xi t 2^{-1 / 3}-i \xi^{3} / 6\right]}{w_{1}^{\prime}(t)}
$$

where the contour $\Gamma_{1}$ runs from infinity to the origin along the ray $\arg t=2 / 3 \pi$, and from the origin to infinity along the positive real axis of $t$.

Combining (47) and (49), $J_{-}(\xi)$ can be given explicity. The main attention will be focussed upon the asymptotic evaluation of $J_{-}(\xi)$ as $|\xi|$ becomes large. This will then yield an asymptotic relationship for $u(y)$ on the flat segment a long distance from the join. Since $J_{+}(\tau)$ has the following asymptotic expression for large $\tau$,

$$
J_{+}(\tau) \sim 2+0\left(\frac{1}{\tau^{3}}\right)
$$

the integral in (47) will be broken up into two portions. The first portion will be of the form

$$
-\frac{e^{-i \pi / 4}}{4} \sqrt{\frac{2}{\pi}} \int_{0}^{\infty} d \tau \frac{\left(\tau^{2}-2 \tau \xi\right)}{(\tau-\xi)^{3 / 2}} \exp \left[\frac{i \tau^{4}}{8(\tau-\xi)}\right]\left\{J_{+}(\tau)-2\right\}
$$

which reduces in the limit $|\xi| \rightarrow \infty$

$$
-\frac{e^{-i \pi / 4}}{2}\left(\frac{2}{\pi|\xi|}\right)^{1 / 2} \int_{0}^{\infty} \tau d \tau\left[J_{+}(\tau)-2\right] .
$$

The second portion is of the form

$$
-\frac{e^{-i \pi / 4}}{2} \sqrt{\frac{2}{\pi}} \int_{0}^{\infty} d \tau\left\{\frac{\left(\tau^{2}-2 \tau \xi\right)}{(\tau-\xi)^{3 / 2}} \exp \left[\frac{i \tau^{4}}{8(\tau-\xi)}\right]\right\}
$$

i.e., it is obtained from the second term on the right-hand side of (47) by replacing $J_{+}(\tau)$ by its asymptotic value 2 corresponding to the physical optics approximation for the current. The above 
expression has the asymptotic value (obtained by setting $\left.\tau=z(-8 \xi)^{1 / 4}\right)$ in the limit as $|\xi| \rightarrow \infty$ :

$$
-e^{-i \pi / 4} \sqrt{\frac{2}{\pi}} \int_{0}^{\infty} \exp \left[i z^{4}\right] d z^{2}+0|\xi|^{-3 / 4},
$$

which reduces to $-1+0|\xi|^{-3 / 4}$. On using (51) and (52), one obtains the following expression for $y \rightarrow \infty$ :

$$
u(y) \sim e^{-i k y}\left[1-(2 \pi|\xi| i)^{-1 / 2} \int_{0}^{\infty} \tau\left[J_{+}(\tau)-2\right] d \tau .\right.
$$

The first term corresponds to the incident field. The second term representing a traveling wave, is the order of $|k y|^{-1 / 2}$.

\section{Discussion}

The results in the preceding two sections can be used separately or together. For instance the surface could be comprised of three segments, two parabolic convex sections smoothly joined to and enclosing a flat section. In this case one convex section would be in the geometric optics illuminated region and the other in the shadow region. If the length $L$ of the flat section is a sufficient number of wavelengths long, then the field distribution on the surface a long distance away from the first joint (the join of the illuminated convex section with the flat section) would be given by (53). Thus the field incident upon the second joint would have amplitude prescribed by (53) with the appropriate value for $\xi$ (i.e., $|y|=L$ ). The field distribution in the shadow region would be prescribed by (35) or (43), modified by the appropriate factor associated with the value of the amplitude and phase of the incident field at the second joint.

Even though the results were derived for cylindrical surfaces, they could be applied locally to a general convex surface provided that the radius of curvature transverse to the direction of incidence was very large compared to wavelength, and the shadow boundary coincides with the join. A special case would be for nose-on incidence to a body of revolution, formed by rotating the profile of the surface about an axis which is parallel to the flat portion of the profile. The results of the second section could be employed to obtain the expression for the creeping waves on the spherical base of a cone-sphere shape where the incident wave would be a wave launched from the tip of the cone.

Finally a few comments should be made regarding the horizontally polarized case. In place of (2), the following is employed for the horizontal polarization:

$$
\frac{\partial u}{\partial n_{P}}=2 \frac{\partial u_{0}}{\partial n_{P}}-\frac{1}{2 \pi} \int \frac{\partial G}{\partial n_{P}} \frac{\partial u}{\partial n_{Q}} d S_{Q}
$$

where $u$ and $\frac{\partial u}{\partial n}$ are proportional respectively to the electric intensity and the surface current (both vectors directed in the $z$ direction). For the geometry treated first wherein the incident wave is a plane wave traveling along the flat portion towards the join with the convex section in the shadow zone, the incident field on the surface (associated with the term $2 \frac{\partial u_{0}}{\partial n}$ ) is zero. Hence the field or current is zero everywhere. A nonvanishing current distribution will be obtained only if the source is included (such a source could be that produced by an edge). For the other geometry where the convex section is in the illuminated zone, the analysis is similar as that for the vertically polarized case. However a long distance away from the junction on the flat portion, the current distribution is just comprised of a traveling wave which decreases in amplitude. 


\section{Appendix $\AA$}

The following integral

$$
F(p)=\int_{0}^{\infty} \exp \left\{-p z-\frac{i z^{3}}{6}\right\} \int_{1}^{\infty}\left(\frac{z}{2}\right)^{3 / 2} \exp \left\{i\left(\frac{z}{2}\right)^{3} \tau\right\} d \tau d z
$$

will be evaluated in terms of Airy integrals. First differentiate the above expression for $F(p)$ twice with respect to $p$ and integrate by parts giving

$$
-\frac{i}{2} F^{\prime \prime}(p)-p F(p)=-\int_{0}^{\infty} \exp \left(i \tau^{2}\right) d \tau+\frac{3}{4 \sqrt{2}} \int_{0}^{\infty} \exp \left(-p z-\frac{i z^{3}}{24}\right) z^{1 / 2} d z
$$

where the prime indicates differentiation with respect to $p$. The first term on the right-hand side is the Fresnel integral and is easily evaluated, and the second integral can be expressed in terms of Airy integrals [Weston, 1962]. The right-hand side thus becomes

$$
-\frac{\sqrt{\pi}}{2} e^{i \pi / 4}+\frac{3}{2} \pi^{3 / 2} e^{i 3 \pi / 4} \frac{d}{d q}\left\{A_{i}(q)\left[A_{i}(q)-i B_{i}(q)\right]\right\}
$$

where the argument $q$ of the Airy integrals are

Set

$$
q=i 2^{1 / 3} p
$$

$$
F(p)=\tilde{F}(t)
$$

The resulting differential equation for $\tilde{F}(t)$ becomes

$$
\frac{d^{2} \tilde{F}(t)}{d t^{2}}-t \tilde{F}(t)=f(t)
$$

where

$$
f(t)=\sqrt{\pi} 2^{-2 / 3} e^{i \pi 5 / 12}\left[-1+3 \pi i \frac{d}{d t}\left\{A_{i}(t)\left[A_{i}(t)+i B_{i}(t)\right]\right\}\right]
$$

is derived upon using the relations

$$
\left.\begin{array}{l}
A_{i}(q)=-\frac{1}{2} e^{2 \pi i / 3}\left[A_{i}(t)+i B_{i}(t)\right] \\
A_{i}(q)-i B_{i}(q)=-2 e^{2 \pi i / 3} A_{i}(t)
\end{array}\right\}
$$

The solution of the homogeneous portion of the differential equation for $\tilde{F}(t)$ are the Airy integrals; hence the complete solution is given by

$$
\tilde{F}(t)=C_{1} A_{i}(t)+C_{2} B_{i}(t)-\pi \int_{0}^{t}\left[A_{i}(t) B_{i}(x)-A_{i}(x) B_{i}(t)\right] f(x) d x
$$

where $C_{1}$ and $C_{2}$ are constants yet to be prescribed. The integral can be reduced using the following relations 


$$
\begin{array}{r}
3 \int_{0}^{t} A_{i}^{2} B_{i}^{\prime} d x=\int_{0}^{t}\left\{\left[2 A_{i} A_{i}^{\prime} B_{i}+\frac{2}{\pi} A_{i}\right]+A_{i}^{2} B_{i}^{\prime}\right\} d x \\
=\frac{2}{\pi} \int_{\neq}^{t} A_{i}(x) d x+\left[A_{i}^{2} B_{i}\right]_{0}^{t} \\
3 \int_{0}^{t} A_{i}^{\prime} A_{i} B_{i} d x=-\frac{1}{\pi} \int_{0}^{t} A_{i}(x) d x+\left[A_{i}^{2} B_{i}\right]_{0}^{t} \\
3 \int_{0}^{t} B_{i}^{\prime} B_{i} A_{i} d x=\frac{1}{\pi} \int_{0}^{t} B_{i}(x) d x+\left[B_{i}^{2} A_{i}\right]_{0}^{t}
\end{array}
$$

giving

$$
\tilde{F}(t)=2^{1 / 3} \pi^{3 / 2} e^{-i \pi / 12}\left[A_{i}(t)+i B_{i}(t)\right] \int_{t}^{\infty} A_{i}(x) d x+C_{1} A_{i}(t)+C_{2} B_{i}(t)
$$

where $C_{1}$ and $C_{2}$ are two new constants.

The next problem is to determine these constants. We shall first consider the behavior as $|t| \rightarrow \infty$ with $|\arg t|<\pi / 3$. In this case

$$
\begin{array}{ll}
A_{i}(t) \sim \frac{1}{2 \sqrt{\pi} t^{1 / 4}} & \exp \left\{-\frac{2}{3} t^{3 / 2}\right\} \\
B_{i}(t) \sim \frac{1}{\sqrt{\pi} t^{1 / 4}} & \exp \left\{\frac{2}{3} t^{3 / 2}\right\}
\end{array}
$$

and $\tilde{F}(t)$ has the asymptotic value

$$
\tilde{F}(t) \sim \frac{\pi^{1 / 2} i}{2^{2 / 3} t} e^{-i \pi / 12}+\frac{C_{2}}{\sqrt{\pi} t^{1 / 4}} \exp \left\{\frac{2}{3} t^{3 / 2}\right\}
$$

The term with coefficient $C_{1}$ is subdominant and can be ignored. Returning to the original expression for $F(p)$ we have, on integrating by parts,

$$
F(p) \sim \frac{1}{p} \int_{0}^{\infty} e^{i \tau^{2}} d \tau+0\left(\frac{1}{p^{2}}\right)
$$

Thus $\tilde{F}(t)$ must have the form for large $|t|$

$$
\tilde{F}(t) \sim \frac{\pi^{1 / 2} i}{2^{2 / 3} t} e^{-i \pi / 12}
$$

and it then is seen that the coefficient $C_{2}$ must vanish.

To evaluate $C_{1}$, we will again let $|t| \rightarrow \infty$ but in the sector $\frac{\pi}{3}<\arg t<2 \pi / 3$ (i.e., $\frac{\pi}{6}<\arg$ $\left.p<\frac{\pi}{2}\right) . \quad$ In this case we shall set

$$
\int_{t}^{\infty} A_{i}(x) d x=\int_{0}^{\infty} A_{i}(x) d x-\int_{0}^{t} A_{i}(x) d x
$$


Setting $t=\tau e^{2 \pi i / 3}$ with $|\arg \tau|<\pi / 3$,

the asymptotic behavior of $A_{1}(t)$ and $B_{1}(t)$ becomes

$$
\begin{gathered}
A_{i}(t)=-\frac{1}{2} e^{4 \pi i / 3}\left[A_{i}(\tau)-i B_{i}(\tau)\right] \sim \frac{-i}{2 \sqrt{\pi} \tau^{1 / 4}} \exp \left\{\pi i / 3+2 / 3 \tau^{3 / 2}\right\} \\
A_{i}(t)+i B_{1}(t)=-2 e^{4 \pi i / 3} A_{i}(\tau) \sim \frac{1}{\sqrt{\pi} \tau^{1 / 4}} \exp \left\{\pi i / 3-2 / 3 \tau^{3 / 2}\right\}
\end{gathered}
$$

It can be shown that

$$
\int_{0}^{t} A_{1}(x) d x \sim \frac{i}{2 \sqrt{\pi} \tau^{3 / 4}} \exp \left\{\frac{2}{3} \tau^{3 / 2}\right\}
$$

hence we have (on neglecting the subdominant terms)

$$
\tilde{F}(t) \sim \frac{\pi^{1 / 2} i e^{-i \pi / 12}}{2^{2 / 3} t}+\frac{C_{1(-i)}}{2 \sqrt{\pi} \tau^{1 / 4}} \exp \left\{\pi i / 3+2 / 3 \tau^{3 / 2}\right\}
$$

and thus it is seen that $C_{1}$ must vanish also.

We have for the final result

$$
\tilde{F}(t)=2^{1 / 3} \pi^{3 / 2} e^{-i \pi / 12}\left[A_{i}(t)+i B_{i}(t)\right]\left\{\int_{0}^{\infty} A_{i}(x) d x-\int_{0}^{t} A_{i}(x) d x\right\} .
$$

The definite integral may be expressed in the form

$$
\int_{0}^{\infty} A_{i}(x) d x=\frac{3^{-1 / 2}}{\pi} \int_{0}^{\infty} K_{1 / 3}(y) d y
$$

involving the modified Hankel function, which reduces to the result

$$
\int_{0}^{\infty} A_{i}(x) d x=\frac{1}{3}
$$

For convenience, (A.19) will be expressed in terms of Airy integrals with argument $q$ given by (A.3). Thus from (A.3) and (A.4) we have

$$
t=e^{2 \pi i / 3} q
$$

and using relationships (A.8), (A.19) becomes

$$
F(p)=e^{i \pi / 4} 2^{4 / 3} \pi^{3 / 2} A_{i}(q)\left\{\frac{1}{3}+\frac{1}{2} \int_{0}^{q}\left[A_{i}(x)-i B_{i}(x)\right] d x\right\},
$$

with $q=-i p 2^{1 / 3}$. 


\section{References}

Fock, V. A. (1946), The distribution of currents induced by a plane wave on the surface of a conductor, J.Phys. 10, No. 2, 130-136.

Keller, J. B., and B. R. Levy (Dec. 1959), Decay exponents and diffraction coefficients for surface waves of nonconstant curvature, IRE Trans. Ant. Prop. AP-7, S52-S61.

Logan, N. A. (Dec. 1959), General research in diffraction theory, 2, Lockheed Missiles and Space Division, 288087.

Maue, A. W. (Aug. 1949), On the formulation of a general diffraction problem through an integral equation, Z. Phys. 126, 601-618.

Miller, J. C. P. (Dec. 1946), The Airy integral, Brit. Assoc. Advancement Science, Math. Tables, Part IIB (University Press, Cambridge).

U.S. National Bureau of Standards (May 1958), Integrals of Airy functions, Appl. Math. Series AMS52.

Weston, V. H. (1962), The effect of a discontinuity in curvature in high frequency scattering, IRE Trans. Ant. Prop. AP-10, 775-780.

(Paper 69D9-561) 